\title{
084
}

\section{LEISHMANIOSI: \\ LA DIAGNOSI DI LABORATORIO}

\author{
Spagnolo P., Spinosa M.
}

Servizio di Immunoematologia e Centro Trasfusionale Day-Hospital Onco-Ematologico,

Presidio Ospedaliero "San Timoteo", Termoli (CB), A.S.L. n.4 Basso-Molise Termoli-Larino.

Introduzione. Diversi microrganismi appartenenti al genere Leishmania parassitano l'uomo.

Si tratta di un gruppo di protozoi in via di differenziazione in seguito all'adattamento a diversi vettori e a diversi ospiti vertebrati.

Nei mammiferi le leishmanie vivono nel citoplasma delle cellule reticolo-endoteliali e si presentano come corpuscoli rotondi o ovoidali allo stadio di amastigoti.

In Italia il vettore principale è il Phlebotomus perniciosus. L'infezione, una tipica zoonosi, ha come serbatoio i cani, che sono una fonte di contagio in quanto le leishmanie si moltiplicano nei tegumenti facilitando l'infezione dei flebotomi. Scopo del nostro lavoro è illustrare la presenza di 5 casi di leishmaniosi viscerale (Kala-azar) accertati presso il nostro Centro negli ultimi 3 anni.

Metodi. La diagnosi di laboratorio si basa sulla dimostrazione diretta del parassita rilevabile nella biopsia midollare mediante colorazione May-Grunwald-Giesma (Sigma). Per la sierodiagnosi abbiamo eseguito il test Leishmania-IgGElisa (Novum Diagnostica).

Risultati. Per i 5 soggetti è stata accertata la presenza del parassita negli strisci di biopsia midollare. È stata rilevata la presenza di IgG anti Leishmania per i 5 soggetti. Inoltre i 5 soggetti presentavano una curva febbrile irregolare, pancitopenia, splenomegalia, anemia, leuco-citopenia, ipergammaglobulinemia, ipoalbuminemia e VES aumentata.

Conclusioni. Il Kala-azar o leishmaniosi viscerale è una malattia grave che in mancanza di trattamento terapeutico può essere letale.

Una parte delle infezioni può manifestarsi clinicamente mentre in alcuni casi il protozoo resta latente per molti anni nei visceri, dando solo positività immunologica. Dopo trattamenti immuodepressivi e nell'AIDS può rilevarsi dopo una latenza pluriennale causando anche lesioni gastriche e intestinali.

Da questi dati emerge la necessità di intraprendere la profilassi, adottando misure contro il randagismo canino, esaminando i cani con conseguente terapia o abbattimento di quelli infetti e per mezzo della lotta contro i flebotomi mediante trattamento con insetticidi. 